\title{
Measles, mumps, rubella and VZV: importance of serological testing of vaccine-preventable diseases in young adults living with HIV in Germany
}

\author{
C. SCHWARZE-ZANDER ${ }^{1,2 * \dagger, ~ R . ~ D R A E N E R T ~}{ }^{3,4} \dagger$, C. LEHMANN $^{2,5}$, \\ M. STECHER ${ }^{2,5}$, C. BOESECKE ${ }^{1,2}$, S. SAMMET ${ }^{3,4}$, J. C. WASMUTH ${ }^{1,2}$, \\ U. SEYBOLD ${ }^{3,4}$ D. GILLOR ${ }^{2,5}$, U. WIELAND ${ }^{6}$, T. KÜMMERLE ${ }^{2,5}$, \\ C. P. STRASSBURG ${ }^{1,2}$, A. MANKERTZ ${ }^{7}$, A. M. EIS-HÜBINGER ${ }^{8}$, G. JÄGER ${ }^{9}$, \\ G. FÄTKENHEUER ${ }^{2,5}$, J. R. BOGNER ${ }^{3,4}$, J. K. ROCKSTROH ${ }^{1,2}$ AND \\ J. J. VEHRESCHILD ${ }^{2,5}$ \\ ${ }^{1}$ Department I for Internal Medicine, University Hospital of Bonn, Bonn, Germany; ${ }^{2}$ German Center for Infection \\ Research (DZIF), partner site Bonn-Cologne, Germany; ${ }^{3}$ Department of Infectious Diseases, Medizinische Klinik und \\ Poliklinik IV, Klinikum der Universität München, Munich, Germany; ${ }^{4}$ German Center for Infection Research \\ (DZIF), partner site Munich, Germany; ${ }^{5}$ Department I for Internal Medicine, University Hospital of Cologne, \\ Cologne, Germany; ${ }^{6}$ Institute of Virology, University Hospital Cologne, Cologne, Germany; ${ }^{7}$ National Reference \\ Center MMR, Robert Koch Institute, Berlin, Germany; ${ }^{8}$ Institute of Virology, University of Bonn, Bonn, Germany; \\ ${ }^{9}$ Institute of Virology, Max von Pettenkofer Institute, Ludwig-Maximilians-University of Munich, Munich, Germany
}

Received 9 March 2016; Final revision 21 August 2016; Accepted 5 September 2016;

first published online 26 October 2016

\section{SUMMARY}

Measles, mumps, rubella (MMR) and varicella zoster virus (VZV) infection can cause serious diseases and complications in the HIV-positive population. Due to successful vaccination programmes measles, mumps and congenital rubella syndrome has become neglected in Germany. However, recent outbreaks of measles have occurred from import-associated cases. In this cross-sectional study the serostatus for MMR and VZV in 2013 HIV-positive adults from three different university outpatient clinics in Bonn $(n=544)$, Cologne $(n=995)$ and Munich $(n=$ 474) was analysed. Sera were tested for MMR- and VZV-specific immunglobulin G antibodies using commercial immunoassays. Seronegativity was found in $3 \%$ for measles, $26 \%$ for mumps, $11 \%$ for rubella and $2 \%$ for VZV. Regarding MMR, 35\% of patients lacked seropositivity against at least one infectious agent. In multivariable analysis younger age was strongly associated with seronegativity against all four viruses, measles, mumps, rubella $(P<0 \cdot 001, P<0.001$ and $P=$ $0 \cdot 001$, respectively) and VZV $(P=0 \cdot 001)$. In conclusion, there is high need for MMR and VZV vaccination in people living with HIV in Germany born in 1970 or later. Thus, systematic MMR and VZV antibody screening and vaccination should be implemented in the HIV-positive population to prevent serious disease and complications of vaccine-preventable diseases.

Key words: HIV, MMR, serology, VZV.

\section{INTRODUCTION}

People living with HIV are more likely to develop serious disease from common vaccine-preventable infections [1]. There has been great concern regarding the safety and efficacy of live-attenuated vaccines in HIV-positive persons. However, studies have shown

\footnotetext{
* Author for correspondence: Dr C. Schwarze-Zander, Medizinische Klinik I, Sigmund-Freud-Str. 25, 53105 Bonn, Germany. (Email: carolynne.schwarze-zander@ukb.uni-bonn.de)

$\uparrow$ These authors contributed equally to this work.
} 
the safety of live-attenuated vaccines against measles, mumps, rubella (MMR) and varicella zoster virus (VZV) in children [2,3] and adults [4-6] living with HIV.

Life expectancy and quality of life have markedly increased in HIV-positive individuals since the introduction of antiretroviral therapy (ART) [7]. Thus, the likelihood of HIV-positive individuals being in contact with vaccine-preventable infections in occupational, social and travel exposures has increased substantially. At the same time, studies from other countries have demonstrated low frequency of seropositivity against MMR or VZV in HIV-positive patients, especially in young adults [1, 8-13]. The guidelines from the European AIDS Clinical Society (EACS) therefore recommend a proactive approach towards screening and vaccination in HIV-positive individuals [14].

While varicella vaccination was recommended for children aged $<24$ months in 2004 [15], the history of MMR vaccination goes back further and differed between former East and West Germany, until in 1991 the MMR combination vaccine was introduced in the reunified Germany. In the former East Germany, measles vaccination was introduced 1970, while mumps and rubella vaccination were not recommended. In the former West Germany, measles vaccination was introduced 1973, followed by measles-mumps vaccination in 1976 and MMR vaccination 1980 [16, 17].

In the absence of a German national registry, standardized data on vaccination coverage for MMR is only obtained at the age of school entry, with rates being consistently $>90 \%$ since 2005 $[18,19]$. However, the aim of the European World Health Organization (WHO) region to eradicate measles and rubella in Europe by 2015 by achieving an overall vaccination coverage of $\geqslant 95 \%$ has not been achieved [20-22]. Introduction of MMR and VZV vaccination into routine childhood immunization programmes has caused a shift of susceptibility, incidence and disease burden towards young adults not having received MMR and VZV vaccination in childhood nor having obtained natural immunity through infection in Germany and other European countries $[19,23]$.

To the best of our knowledge, data regarding the status of seropositivity for these vaccine-preventable diseases are missing in the population living with HIV in Germany. In our cross-sectional study, we therefore aimed to determine the proportion of
HIV-positive adults accessing routine HIV care in Germany who lack seropositivity against MMR and VZV. Furthermore, we attempted to identify demographic, sociological and HIV-related risk factors associated with lack of seroprotection.

\section{METHODS}

\section{Study population}

This study was designed as a cross-sectional multi-centre study. The study was performed at two partner sites of the German Center for Infection Research (DZIF), partner site Bonn-Cologne and partner site Munich. This involved outpatient clinics of three different German university hospitals in Bonn, Cologne, and Klinikum der Universität, LMU, Munich, all treating the full spectrum of HIV infection and associated diseases [24]. Between February 2014 and January 2015 all patients from the outpatient clinics of these three university hospitals were analysed during routine check-up.

Sociodemographic data (age, gender, country of birth, mode of HIV acquisition) and clinical data [duration of HIV infection, US Centers for Disease Control and Prevention (CDC) stage, nadir and baseline $\mathrm{CD}^{+}$lymphocyte count, HIV-1 RNA load, ART] were collected from electronic databases. This study was performed as a substudy of the Translational Platform HIV by the German Centre for Infection Research (NCT02149004) and was approved by the institutional review boards of all sites. All patients from Cologne and Bonn provided written informed consent. The patients from Munich were studied after irreversible de-identification in agreement with the local ethics committee.

\section{Serology}

Serum samples were tested for the presence of $\operatorname{IgG}$ antibodies against MMR and VZV as part of routine patient care. These were tested by means of Siemens Enzygnost anti-measles/IgG, anti-mumps/IgG, antirubella/IgG or anti-VZV/IgG (Siemens, Germany), respectively, with the BEP ${ }^{\circledR}$ III System, except for anti-rubella $\mathrm{IgG}$ in serum samples from Cologne, which was determined with the Architect Rubella IgG Assay (Abbott, Germany).

According to the manufacturers' instructions measles IgG negativity was defined $<150 \mathrm{IU} / 1$, equivocal results as $\geqslant 150$ to $<300 \mathrm{IU} / 1$ and seropositivity as 
$\geqslant 300 \mathrm{IU} / 1$. Mumps IgG negativity was defined as $<230 \mathrm{EU} / \mathrm{ml}$, equivocal results as $\geqslant 230$ to $<460$ $\mathrm{EU} / \mathrm{ml}$ and seropositivity as $\geqslant 460$ EU/l. Rubella IgG negativity was defined $<4$ IU/l (Architect assay $<5 \mathrm{IU} / \mathrm{ml}$ ), equivocal results as $\geqslant 4$ to $<8 \mathrm{IU} / 1$ (Architect assay $\geqslant 5$ to $<10$ ) and seropositivity as $\geqslant 8 \mathrm{IU} / 1$ (Architect assay $\geqslant 10 \mathrm{IU} / \mathrm{ml}$ ). Varicella IgG negativity was defined $<50 \mathrm{IU} / 1$, equivocal results as $\geqslant 50$ to $<100 \mathrm{IU} / 1$ and seropositivity as $\geqslant 100 \mathrm{IU} / 1$. For analysis with dichotomous variables a stringent definition of seropositivity was used and equivocal IgG findings were considered seronegative.

\section{Statistical analysis}

Statistical analyses were completed by using SPSS Statistics software v. 23.0 (IBM Corp., USA). For the assessment of normality, Shapiro-Wilk and Kolmogorov-Smirnov tests were conducted. Normally and not-normally distributed data were analysed by parametric and non-parametric tests, respectively. Characteristics of the patient population were compared by calculation of frequencies, means, and medians. Bivariate analysis using cross-tables and $\chi^{2}$ test with a significance level of $95 \%$ were applied in order to compare characteristics of patients with presence of IgG antibodies against MMR and VZV and those with absence of these antibodies

As measles and MMR vaccination were introduced 1970 and 1980, respectively, the distribution pattern of seropositivity was analysed according to year of birth. All variables were tested for multi-collinearity problems by using correlation matrices (correlation coefficient $<0 \cdot 8$ ) and variance inflation factors (VIF $<10)$ [25]. Binary logistic regression models were used to examine the main effects between variables of interest and the dependent variables' serostatus (negative/ equivocal vs. positive) of MMR and VZV. Odds ratio and $95 \%$ confidence intervals are reported to show the direction and strength of the association (Table 3).

\section{Ethical statement}

This work was performed in accordance with local IRB guidelines of Bonn, Cologne and Munich.

\section{RESULTS}

\section{Study population}

Of the HIV-positive adults attending the HIV outpatient clinics for routine care during the study period,
2013 patients had serum samples taken. There were 544 patients from Bonn, 995 from Cologne, and 474 from Munich (see Table 1).

The majority of these patients were Caucasian $(79 \%)$, male (77\%), and born in Germany $(67 \%)$. Most were receiving ART (89\%) with an undetectable HIV viral load $(79 \%)$ and had median $\mathrm{CD}^{+} \mathrm{T}$ cell counts of 558 cells $/ \mu 1$ [interquartile range (IQR) 390730]. Only $368(18 \%)$ of the 2013 patients had a history of an AIDS-defining event. In the population surveyed, the most common mode of HIV acquisition was men who have sex with men (MSM) (57\%).

\section{Seroprevalence of MMR- and VZV-specific antibodies}

Measles serology was available for 96\% (1937/2013), mumps serology for 96\% (1930/2013), rubella serology for $96 \%$ (1926/2013) and VZV for 97\% (1959/2013) of patients.

The overall distribution of antibodies to MMR and VZV is shown in Table 2. Seropositivity for measles was detected in $92 \%$, for mumps in $74 \%$, for rubella in $90 \%$ and for VZV in $97 \%$ of patients.

Of the $10 \%$ of patients having equivocal values or negative rubella-specific IgG antibodies, 29 were women of childbearing age. Therefore, in this cohort $9 \%$ of women of childbearing age were in need of rubella vaccination with $23 / 29(79 \%)$ women having sufficient $\mathrm{CD}^{+} \mathrm{T}$ cell counts for vaccination $\left(\mathrm{CD} 4^{+}\right.$ $\geqslant 200 / \mu 1$ ).

In Germany, vaccination against MMR is currently only available as a combination vaccine against all three viruses. Thus, we further looked at the distribution pattern of seropositivity against MMR, which was available for 1903 (95\%) of the 2013 patients (Fig. 1). The most frequent pattern was the concomitant prevalence of all three IgG antibody types (measles, mumps, rubella) found in 65\% (1231/1903), while the second most frequent pattern was the prevalence of measles- and rubella-specific antibodies in the absence of mumps antibodies in 19\% (360/1903). Regarding the combination vaccine MMR, 35\% $(672 / 1903)$ of patients had an indication for vaccination against at least one entity, $93 \%$ of these patients having a $\mathrm{CD}^{+} \mathrm{T}$ cell count $\geqslant 200 / \mu 1$, thereby qualifying for vaccination. Considering year of introduction of measles and MMR vaccination we performed an analysis according to year of birth. The most frequent pattern of positive IgG antibodies remained the concomitant prevalence of MMR IgG antibodies; however, the frequency dropped from $71 \%$ for patients 
Table 1. Characteristics of the study population

\begin{tabular}{|c|c|c|}
\hline \multicolumn{3}{|l|}{ Population } \\
\hline Total, $n(\%)$ & 2013 & $(100)$ \\
\hline \multicolumn{3}{|l|}{ Centre, $n(\%)$} \\
\hline Bonn & 544 & $(27)$ \\
\hline Cologne & 995 & (49) \\
\hline Munich & 474 & (24) \\
\hline \multicolumn{3}{|l|}{$N=2013$} \\
\hline Age, years, median (IQR) & 46 & $(39,54)$ \\
\hline \multicolumn{3}{|l|}{$N=2006$} \\
\hline \multicolumn{3}{|l|}{ Year of birth, $n(\%)$} \\
\hline Before 1970 & 1170 & $(58)$ \\
\hline 1970 and after & 838 & (42) \\
\hline$N=2008$ & & \\
\hline \multicolumn{3}{|l|}{ Gender, $n(\%)$} \\
\hline Female & 462 & (23) \\
\hline Male & 1551 & (77) \\
\hline \multicolumn{3}{|l|}{$N=2013$} \\
\hline \multicolumn{3}{|l|}{ Ethnicity, $n(\%)$} \\
\hline Caucasian & 1589 & (79) \\
\hline Afro-American & 287 & (14) \\
\hline Asian & 80 & (4) \\
\hline Hispanic & 40 & (2) \\
\hline \multicolumn{3}{|l|}{$N=1996$} \\
\hline \multicolumn{3}{|l|}{ Country of birth, $n(\%)$} \\
\hline Germany & 1349 & $(67)$ \\
\hline Outside of Germany & 637 & (32) \\
\hline$N=1986$ & & \\
\hline \multicolumn{3}{|l|}{ Mode of HIV acquisition, $n(\%)$} \\
\hline MSM & 1147 & $(57)$ \\
\hline Heterosexual & 388 & (19) \\
\hline IVDU & 73 & (4) \\
\hline High prevalence country & 227 & (11) \\
\hline Other & 178 & (9) \\
\hline$N=2013$ & & \\
\hline $\begin{array}{l}\text { Duration of HIV infection, years, } \\
\text { median (IQR) }\end{array}$ & 8 & $(4,15)$ \\
\hline$N=2008$ & & \\
\hline \multicolumn{3}{|l|}{ CDC stage, $n(\%)$} \\
\hline A & 1434 & $(71)$ \\
\hline $\mathrm{B}$ & 205 & (10) \\
\hline $\mathrm{C}$ & 368 & (18) \\
\hline \multicolumn{3}{|l|}{$N=2007$} \\
\hline $\begin{array}{l}\text { Baseline } \mathrm{CD}^{+} \text {cell count, cells } / \mu \mathrm{l} \text {, } \\
\text { median (IQR) }\end{array}$ & 558 & $(390,730)$ \\
\hline \multicolumn{3}{|l|}{$N=2008$} \\
\hline $\begin{array}{l}\text { Nadir CD4 }{ }^{+} \text {cell count, cells } / \mu 1, \\
\text { median }(\mathrm{IQR})\end{array}$ & 200 & $(70,330)$ \\
\hline$N=1976$ & & \\
\hline \multicolumn{3}{|l|}{ HIV-RNA, $n(\%)$} \\
\hline$\leqslant 40$ copies $/ \mathrm{ml}$ & 1583 & (79) \\
\hline$>40$ copies $/ \mathrm{ml}$ & 423 & (21) \\
\hline$N=2006$ & & \\
\hline
\end{tabular}

\begin{tabular}{cl} 
ART, $n(\%)$ & 1793 \\
On ART & $(89)$ \\
No ART & 218 \\
$N=2011$ & \\
\hline
\end{tabular}

IQR, Interquartile range; MSM, men who have sex with men; IVDU, intravenous drug use; CDC, US Centers for Disease Control and Prevention; ART, antiretroviral therapy; $N$, number of patients available for respective parameter.

Table 2. Frequency of positive, equivocal and negative measles, mumps, rubella and VZVIgG antibodies in people living with HIV in Germany

\begin{tabular}{llcc}
\hline \hline & Positive, \% $(n)$ & Equivocal, \% $(n)$ & Negative, \% $(n)$ \\
\hline Measles & $92(1789)$ & $4(86)$ & $3(62)$ \\
Mumps & $74(1421)$ & $13(257)$ & $13(252)$ \\
Rubella & $90(1724)$ & $3(56)$ & $8(146)$ \\
VZV & $97(1896)$ & $1(17)$ & $2(46)$ \\
\hline \hline
\end{tabular}

born $1930-1969$, to $58 \%$ for patients born $1970-1979$ and $49 \%$ in patients born $1980-1995$.

Furthermore, serostatus of MMR and VZV was analysed according to the sociodemographic and clinical patients' data (for detailed overview see Supplementary Table S1 $a-d$ ). By bivariate analysis, for all viruses seropositivity declined with younger age (Supplementary Table S1 $a-d$, Fig. 2). Furthermore, seropositivity for measles was lower in patients with a shorter duration of HIV infection (mean 8 years in negative $v s .10$ years in positive, $P=0.007$ ), replicating HIV viral load $(3 \%$ measles IgG negative with HIV-RNA $<40$ copies $/ \mathrm{ml}$ vs. $5 \%$ measles IgG negative with HIV-RNA $\geqslant 40$ copies $/ \mathrm{ml}, P=0.03$ ). In addition an association of measles serostatus and CDC stage was found ( $4 \%$ measles $\mathrm{IgG}$ negative in CDC A, $0 \%$ in CDC B and $3 \%$ in CDC C, $P=0.017$ ). For mumps seropositivity was lower in patients born in Germany $(15 \%$ mumps $\operatorname{IgG}$ negative born in Germany vs. 9\% mumps IgG negative born outside Germany, $P=0.002)$ and in MSM (15\% mumps IgG negative in MSM vs. $10 \%$ mumps IgG negative in non-MSM, $P=0.001)$. Seropositivity for rubella was lower in patients with baseline $\mathrm{CD}^{+}<200 / \mu \mathrm{l}$ (13\% rubella $\mathrm{IgG}$ negative with $\mathrm{CD}^{+}<200 / \mu 1$ vs. 
Table 3. Logistic regression model of main effects between variables of interest and measles, mumps, rubella and VZV IgG seronegativity/equivocal

\begin{tabular}{|c|c|c|c|c|c|c|c|c|}
\hline & \multicolumn{2}{|c|}{ Measles IgG negative* } & \multicolumn{2}{|c|}{ Mumps IgG negative* } & \multicolumn{2}{|l|}{ Rubella IgG negative* } & \multicolumn{2}{|l|}{ VZV IgG negative* } \\
\hline & OR $(95 \% \mathrm{CI})$ & $P$ & OR $(95 \% \mathrm{CI})$ & $P$ & OR $(95 \% \mathrm{CI})$ & $P$ & OR $(95 \% \mathrm{CI})$ & $P$ \\
\hline Country of birth: Germany & ** & & $1.439(1 \cdot 111-1 \cdot 863)$ & 0.006 & $1.409(1.003-1 \cdot 980)$ & 0.048 & $* *$ & \\
\hline Duration of HIV infection (years) & ** & & $1 \cdot 013(1.005-1 \cdot 022)$ & 0.002 & $* *$ & & $1 \cdot 022(1 \cdot 001-1 \cdot 044)$ & 0.040 \\
\hline HIV acquisition: MSM & ** & & $1.320(1.044-1.669)$ & $0 \cdot 020$ & ** & & ** & \\
\hline \multicolumn{9}{|l|}{ CDC stage (ref. stage C) } \\
\hline A & $0.853(0.510-1.426)$ & 0.543 & $0.738(0.537-1 \cdot 015)$ & $0 \cdot 062$ & $* *$ & & $* *$ & \\
\hline B & $0.289(0.096-0.872)$ & 0.028 & $1.138(0.757-1.711)$ & 0.533 & ** & & ** & \\
\hline \multicolumn{9}{|l|}{ CD4 nadir $(\operatorname{cells} / \mu 1)($ ref. $\geqslant 500)$} \\
\hline$<200$ & ** & & $* *$ & & $2.036(0 \cdot 979-4 \cdot 235)$ & $0 \cdot 057$ & $* *$ & \\
\hline$<350$ & ** & & ** & & $1.799(0.868-3.728)$ & $0 \cdot 114$ & ** & \\
\hline$<500$ & ** & & ** & & $1.573(0.716-3.456)$ & $0 \cdot 260$ & ** & \\
\hline \multicolumn{9}{|l|}{ CD4 baseline $(\operatorname{cells} / \mu 1)($ ref. $\geqslant 500)$} \\
\hline$<200$ & $1.796(0 \cdot 900-3.586)$ & 0.097 & $1 \cdot 218(0.743-2.023)$ & $0 \cdot 446$ & $1 \cdot 768(0.952-3 \cdot 284)$ & $0 \cdot 071$ & ** & \\
\hline$<350$ & $1.673(0.988-2 \cdot 832)$ & $0 \cdot 055$ & $1.007(0.716-1.417)$ & 0.967 & $1.236(0.778-1.965)$ & $0 \cdot 370$ & ** & \\
\hline$<500$ & $1.104(0.693-1.760)$ & 0.676 & $0.716(0.533-0.961)$ & 0.026 & $0.940(0.623-1.418)$ & $0 \cdot 767$ & ** & \\
\hline HIV RNA (copies/ml) $\leqslant 40$ & ** & & $0.806(0.604-1 \cdot 076)$ & 0.233 & $0.828(0.540-1 \cdot 271)$ & $0 \cdot 388$ & $1 \cdot 022(1 \cdot 001-1 \cdot 044)$ & 0.045 \\
\hline On ART & $* *$ & & ** & & $1.308(0.681-2.512)$ & $0 \cdot 420$ & ** & \\
\hline Age (years) & $0.909(0.892-0.927)$ & $0 \cdot 000$ & $0.961(0.950-0.972)$ & $0 \cdot 000$ & $0.976(0.962-0.990)$ & $0 \cdot 001$ & $0.942(0 \cdot 913-0.968)$ & 0.000 \\
\hline Male sex & $1.134(0.850-2.030)$ & 0.219 & ** & & $* *$ & & $0.637(0.358-1.133)$ & $0 \cdot 125$ \\
\hline
\end{tabular}

OR, Odds ratio; CI, confidence interval; MSM, men who have sex with men; CDC, US Centers for Disease Control and Prevention; ART, antiretroviral therapy.

* Equivocals are grouped with the seronegatives. The reference category is positive.

** These variables were deleted during backward elimination. 


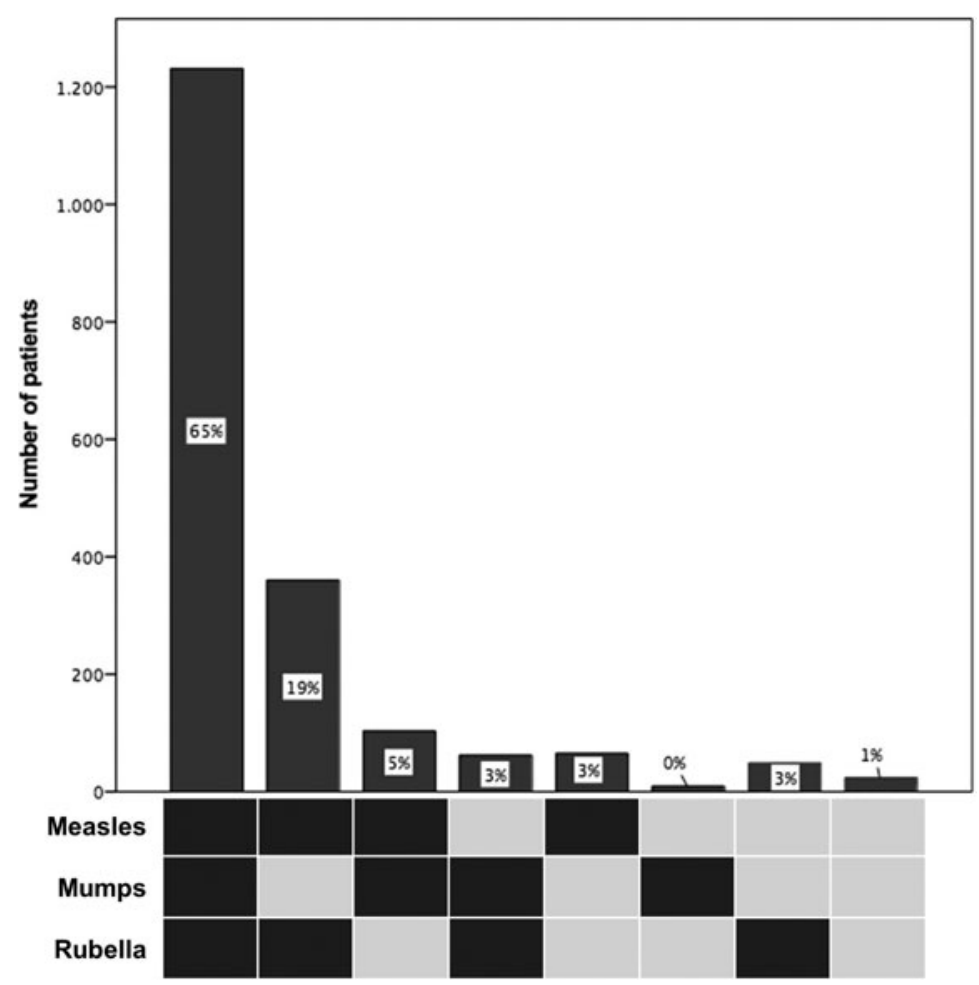

Fig. 1. Distribution patterns of measles, mumps and rubella protective IgG antibody combinations in 1903 HIV patients. Dark squares indicate seropositivity.

$7 \%$ rubella $\mathrm{IgG}$ negative with $\mathrm{CD}^{+} \geqslant 200 / \mu \mathrm{l}$, $P=0.02)$. Finally, seropositivity for varicella was lower in male patients ( $2 \%$ varicella $\mathrm{IgG}$ negative in females $v s .3 \cdot 6 \%$ varicella $\mathrm{IgG}$ negative in males, $P=0.026)$ and an association was found with CDC stage $(3 \%$ varicella $\mathrm{IgG}$ negative $\mathrm{CDC} \mathrm{A}, 2 \%$ in CDC B and $1 \%$ CDC C, $P=0.04$ ).

We furthermore calculated a binary logistic regression model for MMR and VZV serostatus identifying and comparing factors predicting seronegativity/ equivocal. Variables showing association $(P \leqslant 0.25)$ with serostatus of at least one of the four viruses were offered to the model.

Our final model included the clinically defined risk factors country of birth, duration of HIV infection, $\mathrm{CDC}$ stage, nadir $\mathrm{CD}^{+}$cell count, baseline $\mathrm{CD}^{+}$ cell count, HIV RNA, ART status, age and gender. For all four viruses, age was a significant factor of seronegativity/equivocal. None of the models was able to properly predict seronegativity, with pseudo$R^{2}$ values ranging from 0.059 to 0.163 (Table 3 ). These results were very robust towards sensitivity analysis, including backward and forward elimination for selection of variables. This model revealed some significant associations for country of birth (mumps and rubella), duration of HIV infection (mumps and
VZV), HIV acquisition (mumps), CDC stage (measles), CD4 baseline (mumps) and HIV RNA (VZV) (for detailed overview see Table 3).

\section{DISCUSSION}

In our cross-sectional study of adults living with HIV in Germany and accessing routine care, we found as many as $35 \%$ of patients susceptible to at least one infection preventable by MMR vaccination and $2 \%$ of patients susceptible to VZV.

Our analysis revealed an overall low frequency of measles seronegativity $(3 \cdot 2 \%)$ in the 1937 adult HIVpositive patients screened for measles antibodies. This result is consistent with studies performed in other European countries, such as France $(4.6 \%)$ [10], Austria (8\%) [12], UK (7\%) [1] and Spain (5\%) [13]. However, we found a strong independent association between measles serostatus and age. While the frequency of measles seronegativity was lowest $(0 \%)$ in patients born between 1930 and 1940, it continuously increased through decades of birth, reaching as high as $26 \%$ between 1990 and 1995 . This observation probably relates to emerging vaccination programmes disrupting transmission of natural infection, thus increasing frequency of seronegative patients in the 

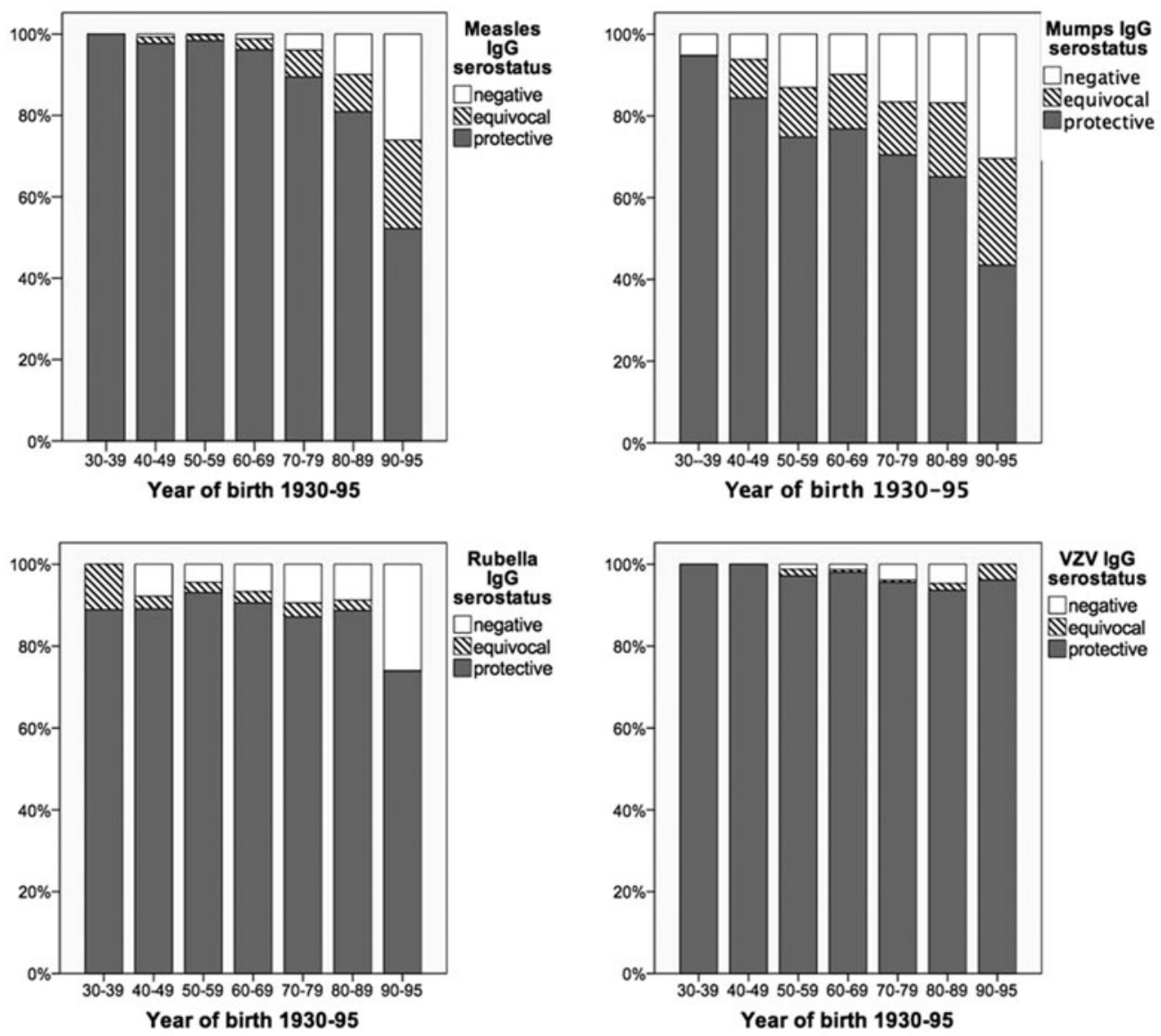

Fig. 2. Frequency of negative, equivocal and positive measles, mumps, rubella and VZV IgG antibodies by year of birth (1930-1995).

group of non-vaccinated patients and vaccination non-responders. In HIV patients born outside Germany, the frequency of measles seronegativity did not differ from that of patients born in Germany, which is in line with results from studies performed in France [10] and Spain [13].

Measles has seen a marked resurgence during recent years in Germany, with the latest outbreak in Berlin affecting >1000 individuals between October 2014 and May 2015 [26]. In agreement with our data on lack of seropositivity to measles especially in individuals born between 1970 and 1995, around one third of persons with measles infection in the recent outbreak were aged 20-40 years [26].

In our study, $26 \%$ and $11 \%$ of HIV-positive adults were susceptible to mumps and rubella virus infection, respectively. There is no evidence suggesting a more severe course of mumps or rubella in HIV-positive individuals. However, especially in women of childbearing age, we found $9 \%$ had no seropositivity against rubella, thus risking severe complications of rubella infection in case of pregnancy. In our study, $79 \%$ of these had $\mathrm{CD}^{+} \mathrm{T}$ cell counts $\geqslant 200 / \mu \mathrm{l}$ and were potentially eligible for vaccination.

We found $<3 \%$ of HIV-positive adults negative for VZV-specific IgG antibodies, which again was associated with decade of birth. Given HIV-positive patients are at increased risk of complications and severe courses of VZV infection, HIV patients lacking VZV seropositivity should be evaluated for varicella vaccination.

Recently, an epidemiological report on the seroprevalence of MMR antibodies in children and adolescents aged between 1 and 17 years living in Germany described an increase of seronegativity by years since last vaccination, indicating a significant antibody waning effect for all three components of the MMR vaccine [16]. This is supported by a study investigating the seropositivity for MMR and VZV in 223 medical students with a median age of $23 \cdot 4$ years in Germany [27]. Seropositivity was detected in $91 \cdot 5 \%$ for measles, $80 \cdot 3 \%$ for mumps, $90 \cdot 1 \%$ for 
rubella and $96.9 \%$ for varicella, demonstrating a high need for immunization in this age group. HIV status seems to play a minor role in the significant gap of seropositivity found in young adults. This may be explained by the fact, that immunity gained by natural infection and vaccination-acquired immunity are developed long before HIV infection occurs. HIV infection does not seem to significantly alter MMR and varicella zoster seropositivity acquired before HIV infection.

There are limitations to this study. The vaccination history was not evaluated as records of vaccination history are incomplete. Thus, we are unable to make conclusions concerning vaccination history and current seropositivity of MMR- and VZV-specific antibodies. Time of immigration into Germany has not been recorded, possibly influencing seropositivity for MMR and VZV in patients born outside Germany. Furthermore, no direct conclusion can be made on immunity against these viruses, as we restricted our study to measuring serum IgG levels in ELISAs.

The guidelines from the EACS [14] recommend screening for antibodies against vaccine-preventable diseases, such as MMR and VZV, at initial and subsequent visits of HIV-positive persons. Vaccination of those that are seronegative is recommended if the $\mathrm{CD}^{+}{ }^{+} \mathrm{T}$ cell count is $\geqslant 200$ cells $/ \mu \mathrm{l}$. It is unclear how strictly these recommendations are followed by HIV physicians across the European region. Our data support that screening and subsequent vaccination must be implemented more widely in Germany especially in HIV-positive individuals born in 1970 or later.

In conclusion, we found $35 \%$ of HIV-positive individuals in need of MMR vaccination and $2 \%$ in need of varicella vaccination. While HIV-related factors seem to play a minor role, being born in 1970 or later is associated with risk of seronegativity for MMR and VZV, all four being vaccine-preventable diseases. Considering the recent measles outbreak in Berlin, our study provides useful data on the importance of consequently implementing routine serological screening for vaccine-preventable diseases in the HIVpositive population. Vaccination remains the cornerstone of disease prevention.

\section{SUPPLEMENTARY MATERIAL}

For supplementary material accompanying this paper visit http://dx.doi.org/10.1017/S095026881600217X.

\section{ACKNOWLEDGEMENTS}

We thank all the technicians of the Institutes of Virology from Bonn, Cologne and Munich for supporting this work. This work was partly funded by the Federal Ministry of Research (BMBF) through the Deutsches Zentrum für Infektionsforschung (DZIF).

\section{DECLARATION OF INTEREST}

None.

\section{REFERENCES}

1. Molton J, et al. Seroprevalence of common vaccine-preventable viral infections in HIV-positive adults. Journal of Infection 2010; 61: 73-80.

2. Scott $\mathbf{P}$, et al. Measles vaccination in HIV-infected children: systematic review and meta-analysis of safety and immunogenicity. Journal of Infectious Diseases 2011; 204 (Suppl. 1): S164-178.

3. Helfand RF, et al. Evaluation of the immune response to a 2-dose measles vaccination schedule administered at 6 and 9 months of age to HIV-infected and HIV-uninfected children in Malawi. Journal of Infectious Diseases 2008; 198: 1457-1465.

4. Stermole BM, et al. Long-term safety and serologic response to measles, mumps, and rubella vaccination in HIV-1 infected adults. Vaccine 2011; 29: 2874-2880.

5. Belaunzaran-Zamudio PF, et al. Early loss of measles antibodies after MMR vaccine among HIV-infected adults receiving HAART. Vaccine 2009; 27: 7059-7064.

6. Wallace MR, et al. Measles seroprevalence and vaccine response in HIV-infected adults. Vaccine 1994; 12: 1222-1224.

7. Ehren K, et al. Causes of death in HIV-infected patients from the Cologne-Bonn cohort. Infection 2014; 42: 135-140.

8. Kemper CA, et al. The prevalence of measles antibody in human immunodeficiency virus-infected patients in northern California. Journal of Infectious Diseases 1998; 178: 1177-1180.

9. Kemper CA, et al. Prevalence of measles antibodies in adults with HIV infection: possible risk factors of measles seronegativity. Aids 1992; 6: 1321-1325.

10. Lambert D, et al. High prevalence of measles seronegativity in adults with HIV infection born in the era of measles vaccination in northern France. Aids 2015; 29: 241-243.

11. Choudhury SA, et al. Immunity to measles in pregnant mothers and in cord blood of their infants: Impact of HIV status and mother's place of birth. Journal of the National Medical Association 2008; 100: 1445-1449.

12. Grabmeier-Pfistershammer $\mathbf{K}$, et al. High need for MMR vaccination in HIV infected adults in austria. Vaccine 2014; 32: 6020-6023.

13. Llenas-Garcia J, et al. Do HIV-positive adult immigrants need to be screened for measles-mumps-rubella 
and varicella zoster virus immunization? AIDS Care 2013; 25: 980-989.

14. EACS. Guidelines version 7.1. European AIDS Clinical Society, 2014.

15. Robert Koch-Institut. Varicella vaccination [in German]. Epidemiologisches Bulletin 2004; 49: 421-432.

16. Poethko-Muller C, Mankertz A. Seroprevalence of measles-, mumps- and rubella-specific IgG antibodies in German children and adolescents and predictors for seronegativity. PLOS ONE 2012; 7: e42867.

17. Klein S, Schöneberg I, Krause G. The historical development of immunization in Germany. From compulsory smallpox vaccination to a National Action Plan on Immunization [in German]. Bundesgesundheitsblatt 2012; 55: 1512-1523.

18. Robert Koch-Institut. Vaccination rate at time of school entry in Germany, 2005 [in German] (http://www.rki.de/ DE/Content/Infekt/Impfen/Impfstatus/schulanfaenger/ ImpfstatusDeutschland2005.pdf?_blob=publicationFile).

19. Robert Koch-Institut. Vaccination rate at time of school entry in Germany, 2013 [in German]. Epidemiologisches Bulletin 2015; 16: 131-138.

20. World Health Organization. Eliminating measles and rubella - framework for the verification process in the WHO European region. WHO Regional Office for Europe, 2014.
21. Santibanez $\mathbf{S}$, et al. Long-term transmission of measles virus in central and continental western Europe. Virus Genes 2015; 50: 2-11.

22. Santibanez S, Mankertz A. Molecular surveillance shows progress in measles elimination process [in German]. Bundesgesundheitsblatt, Gesundheitsforschung, Gesundheitsschutz 2013; 56: 1238-1242.

23. Corbin V, et al. Shift of the 2009-2011 measles outbreak from children to adults: an observational review at the university hospital of Clermont-Ferrand, France. Infection 2013; 41: 1157-1161.

24. Rockstroh JK. The Cologne-Bonn cohort: lessons learned. Infection 2015; 43: 135-139.

25. Yu H, Jiang S, Land KC. Multicollinearity in hierarchical linear models. Social Science Research 2015; 53: 118-136.

26. Robert Koch-Institut. Overview on the epidemiology of measles in 2014 and current situation in 2015 in Germany [in German]. Epidemiologisches Bulletin 2015; 10: 69-74.

27. Wicker S, et al. Seroprevalence of vaccine preventable and blood transmissible viral infections (measles, mumps, rubella, polio, HBV, HCV and HIV) in medical students. Medical Microbiology and Immunology 2007; 196: $145-150$. 